\title{
Spectral analysis of the subelliptic oblique derivative problem
}

\author{
Kazuaki Taira
}

Dedicated to the memory of Professor Louis Boutet de Monvel (1941-2014)

\begin{abstract}
This paper is devoted to a functional analytic approach to the subelliptic oblique derivative problem for the usual Laplacian with a complex parameter $\lambda$. We solve the long-standing open problem of the asymptotic eigenvalue distribution for the homogeneous oblique derivative problem when $|\lambda|$ tends to $\infty$. We prove the spectral properties of the closed realization of the Laplacian similar to the elliptic (non-degenerate) case. In the proof we make use of Boutet de Monvel calculus in order to study the resolvents and their adjoints in the framework of $L^{2}$ Sobolev spaces.
\end{abstract}

\section{Formulation of the oblique derivative problem}

Due to Poincaré [19], it is known that the oblique derivative problem arises naturally when determining the gravitational field of the moon, the earth and the other celestial bodies.

In physical geodesy, investigations of the Earth's gravity field based on surface gravity data are usually associated with a simultaneous determination of the figure of the Earth. The precise 3D positioning by the Global Navigation Satellite Systems has brought new possibilities in gravity field modelling. Terrestrial gravimetric measurements located by precise satellite positioning yield oblique derivative boundary conditions in the form of surface gravity disturbances. Now the shape of the Earth can be obtained by geometric satellite triangulation and satellite altimetry over the oceans. In this way, the (linearized) fixed gravimetric boundary value problem in

Key words and phrases: oblique derivative problem, subelliptic operator, asymptotic eigenvalue distribution, Boutet de Monvel calculus.

2010 Mathematics Subject Classification: 35J25, 35S05, 47D03, 35P20. 
physical geodesy is an oblique derivative problem for the Laplace equation in the Earth's exterior, where the physical surface of the Earth is assumed to be known (see [4] and [16]).

In this paper we will deal with an interior oblique derivative problem in a bounded domain. It should be noticed that the analysis of harmonic functions in an exterior domain can be reduced to that of harmonic functions in a bounded domain by using the Kelvin transform, called the inverse radii transform (see [3, Chapter 4]).

Let $\Omega$ be a bounded domain of Euclidean space $\mathbf{R}^{n}, n \geq 3$, with smooth boundary $\Gamma=\partial \Omega$; its closure $\bar{\Omega}=\Omega \cup \Gamma$ is an $n$-dimensional, compact smooth manifold with boundary $\Gamma$. In this paper, for the usual Laplacian

$$
\Delta=\sum_{i=1}^{n} \frac{\partial^{2}}{\partial x_{i}^{2}}=\frac{\partial^{2}}{\partial x_{1}^{2}}+\frac{\partial^{2}}{\partial x_{2}^{2}}+\ldots+\frac{\partial^{2}}{\partial x_{n}^{2}},
$$

we consider an oblique derivative boundary condition $B \boldsymbol{\gamma}$ such that

$$
B \gamma u=\frac{\partial u}{\partial \boldsymbol{\nu}}=a\left(x^{\prime}\right) \frac{\partial u}{\partial \mathbf{n}}+\alpha\left(x^{\prime}\right) \cdot u
$$

Here:

(1) $a \in C^{\infty}(\Gamma)$.

(2) $\alpha\left(x^{\prime}\right)$ is a real, smooth tangential vector field on $\Gamma$. More precisely, in terms of a local coordinate system $\left(x_{1}, x_{2}, \ldots, x_{n-1}\right)$ of $\Gamma$, the vector field $\alpha\left(x^{\prime}\right)$ has the local expression

$$
\alpha\left(x^{\prime}\right)=\sum_{k=1}^{n-1} \alpha_{k}\left(x^{\prime}\right) \frac{\partial}{\partial x_{k}} .
$$

(3) $\boldsymbol{\nu}=a\left(x^{\prime}\right) \mathbf{n}+\alpha\left(x^{\prime}\right)$ is a smooth, nowhere vanishing vector field on $\Gamma$ where $\mathbf{n}=\left(n_{1}, n_{2}, \ldots, n_{n}\right)$ is the unit outward normal to $\Gamma$.

We study the following homogeneous oblique derivative problem: Given a function $f(x)$ defined in $\Omega$, find a function $u(x)$ in $\Omega$ such that

$$
\begin{cases}(\Delta-\lambda) u=f & \text { in } \Omega, \\ B \gamma u=a\left(x^{\prime}\right) \frac{\partial u}{\partial \mathbf{n}}+\alpha\left(x^{\prime}\right) \cdot u=0 & \text { on } \Gamma,\end{cases}
$$

where $\lambda$ is a complex parameter.

We remark that the oblique derivative problem (1.1) is non-degenerate (or coercive) if and only if $a\left(x^{\prime}\right) \neq 0$ on $\Gamma$, that is, the vector field $\boldsymbol{\nu}=a\left(x^{\prime}\right) \mathbf{n}+\alpha\left(x^{\prime}\right)$ is nowhere tangent to $\Gamma$ (see [9] and [13]). 


\section{Statement of main result}

Our starting point is to state an existence and uniqueness theorem of the subelliptic oblique derivative problem (1.1) in the framework of $L^{2}$ Sobolev spaces when $|\lambda|$ tends to $\infty$, due to $[29$, Theorem 2.2]:

Theorem 2.1. Assume that the following hypothesis $(\mathrm{H})$ is satisfied:

$(\mathrm{H})$ The vector field $\alpha\left(x^{\prime}\right)$ is non-zero on the set $\Gamma_{0}=\left\{x^{\prime} \in \Gamma: a\left(x^{\prime}\right)=0\right\}$ of tangency and, along the integral curve $x\left(t, x_{0}^{\prime}\right)$ of $\alpha\left(x^{\prime}\right)$ passing through $x_{0}^{\prime} \in \Gamma_{0}$ at $t=0$, the function: $t \mapsto a\left(x\left(t, x_{0}^{\prime}\right)\right)$ has zeros of even order $\leq 2 k$ for some non-negative integer $k$.

Then, for every $\theta \in(-\pi, \pi)$ there exists a constant $R(\theta)>0$, depending on $\theta$, such that if $\lambda=r^{2} e^{i \theta}$ satisfies the condition $|\lambda|=r^{2} \geq R(\theta)$, the homogeneous oblique derivative problem (1.1) has a unique solution $u \in H^{2}(\Omega)$ for any $f \in L^{2}(\Omega)$. Moreover, we have the a priori estimate

$$
\|u\|_{H^{2}(\Omega)}+|\lambda|\|u\|_{L^{2}(\Omega)} \leq C(\theta)\|f\|_{L^{2}(\Omega)},
$$

with a constant $C(\theta)>0$ depending on $\theta$.

Here and in the following $H^{s}(\Omega)=W^{s, 2}(\Omega)$ denotes the $L^{2}$ Sobolev space of order $s$ on $\Omega$.

Remark 2.2. The hypothesis $(\mathrm{H})$ implies that the function $a\left(x^{\prime}\right)$ does not change sign on the boundary $\Gamma$. This is called Case I in Guan-Sawyer [11]. Moreover, it is easy to see that the hypothesis $(\mathrm{H})$ is equivalent to saying that the vector field $\boldsymbol{\nu}$ is of finite type on $\Gamma$ defined in Smith [23] and Guan-Sawyer [11].

The elliptic estimate (2.1) for a degenerate problem works, since we are considering the homogeneous boundary condition. On the other hand, we can give a necessary and sufficient condition in order that the non-homogeneous oblique derivative problem

$$
\begin{cases}(\Delta-\lambda) u=f & \text { in } \Omega, \\ B \gamma u=\varphi & \text { on } \Gamma\end{cases}
$$

is subelliptic in the framework of $L^{2}$ Sobolev spaces (see [25, Théorème 11]).

Now we associate with the homogeneous oblique derivative problem (1.1) a densely defined, closed linear operator

$$
\mathfrak{A}_{2}: L^{2}(\Omega) \longrightarrow L^{2}(\Omega)
$$

in the Hilbert space $L^{2}(\Omega)$ as follows (see Claim 8.1 for $p:=2$ ):

(a) The domain $\mathcal{D}\left(\mathfrak{A}_{2}\right)$ of definition is the space

$$
\mathcal{D}\left(\mathfrak{A}_{2}\right)=\left\{u \in H^{2}(\Omega): B \gamma u=0 \text { on } \Gamma\right\} .
$$

(b) $\mathfrak{A}_{2} u=\Delta u$ for every $u \in \mathcal{D}\left(\mathfrak{A}_{2}\right)$. 
In this paper, by combining Agmon [2, Theorems 14.4 and 15.1] with Theorem 2.1 we shall prove the following spectral properties of the closed realization $\mathfrak{A}_{2}$ of $\Delta$ similar to the elliptic (non-degenerate) case:

Theorem 2.3. Assume that the hypothesis $(\mathrm{H})$ is satisfied. Then the operator $\mathfrak{A}_{2}$ enjoys the following five spectral properties:

(i) The spectrum of $\mathfrak{A}_{2}$ is discrete and the eigenvalues $\lambda_{j}$ of $\mathfrak{A}_{2}$ have finite multiplicities.

(ii) All rays $\arg \lambda=\theta$ different from the negative axis are rays of minimal growth of the resolvent $\left(\mathfrak{A}_{2}-\lambda I\right)^{-1}$. In fact, we have, by estimate (2.1),

$$
\left\|\left(\mathfrak{A}_{2}-\lambda I\right)^{-1}\right\| \leq \frac{C(\theta)}{|\lambda|} \quad \text { for all }|\lambda| \geq R(\theta) .
$$

(iii) The negative axis is a direction of condensation of eigenvalues of $\mathfrak{A}_{2} ;$ more precisely, for each $\varepsilon>0$ there are only a finite number of eigenvalues outside the angle: $-\pi+\varepsilon<\theta<\pi-\varepsilon$.

(iv) Let

$$
N(t):=\sum_{\operatorname{Re} \lambda_{j} \geq-t} 1
$$

be the number of eigenvalues $\lambda_{j}$ such that $\operatorname{Re} \lambda_{j} \geq-t$, where each $\lambda_{j}$ is repeated according to its multiplicity. Then the asymptotic eigenvalue distribution formula

$$
N(t)=\frac{|\Omega|}{2^{n} \pi^{n / 2} \Gamma(n / 2+1)} t^{n / 2}+o\left(t^{n / 2}\right) \quad \text { as } t \rightarrow+\infty
$$

holds true. Here $|\Omega|$ denotes the volume of the domain $\Omega$.

(v) The generalized eigenfunctions of $\mathfrak{A}_{2}$ are complete in the Hilbert space $L^{2}(\Omega)$; they are also complete in the domain $\mathcal{D}\left(\mathfrak{A}_{2}\right)$ in the $H^{2}(\Omega)$-norm.

Theorem 2.3 solves the long-standing open problem of the asymptotic eigenvalue distribution for the subelliptic oblique derivative problem (1.1). Theorem 2.3 (and Theorem 10.1) was announced in the previous paper [29, Corollary 2.3].

The rest of this paper is organized as follows. In Section 3 we formulate a characterization of classical subelliptic pseudo-differential operators due to Egorov [8] and Hörmander [15] (Theorem 3.1) which plays a crucial role in this paper. In Section 4 we consider non self-adjoint eigenvalue problems for a general secondorder, uniformly elliptic differential operator $A$. In particular, we characterize some spectral properties of the closed realization $\mathcal{A}$ of $A$ in terms of their resolvents 
(Theorem 4.1), essentially due to Agmon [2, Theorems 14.4 and 15.1]. In Section 5 we prove that an oblique derivative problem

$$
\begin{cases}\Delta u=f & \text { in } \Omega, \\ B \gamma u=0 & \text { on } \Gamma\end{cases}
$$

can be reduced to the study of a pseudo-differential operator $T$ on the boundary $\Gamma$ (Proposition 5.2). The virtue of this reduction is that there is no difficulty in taking adjoints or transposes after restricting the attention to the boundary, whereas boundary value problems in general do not have adjoints or transposes. In Subsection 5.1 we study the oblique derivative boundary condition $B$ in the framework of $L^{p}$ Sobolev spaces. In Subsection 5.2 we show precisely that the oblique derivative problem (2.3) can be reduced to the study of the pseudo-differential operator $T$ on $\Gamma$ in the framework of $L^{p}$ Sobolev spaces (Theorem 5.6). In Subsection 5.3 we prove that if condition $(\mathrm{H})$ is satisfied, then the index of the oblique derivative problem (2.3) is independent of $p$ for all $1<p<\infty$ (Theorem 5.6). In Section 6 we prove a uniqueness theorem for the non-homogeneous oblique derivative problem

$$
\begin{cases}(1-\Delta) u=f & \text { in } \Omega, \\ B \gamma u=\varphi & \text { on } \Gamma\end{cases}
$$

in the framework of $L^{p}$ Sobolev spaces (Theorem 6.1). In Section 7, by using the Poisson kernel of $1-\Delta$ in the exterior domain of $\Omega$ we reduce the homogeneous oblique derivative problem (2.4) to the study of a first-order, pseudo-differential operator $T$ on the boundary $\Gamma$ (Proposition 7.1), just as in Smith [23] and GuanSawyer [11]. In Section 8 we can express explicitly the unique solution $u$ of the homogeneous oblique derivative problem

$$
\begin{cases}(1-\Delta) u=f & \text { in } \Omega, \\ B \gamma u=0 & \text { on } \Gamma .\end{cases}
$$

Moreover, we can characterize the regularity property of the resolvents for the oblique derivative problem (2.5) (Corollary 8.2). In Section 9 we prove Theorem 2.3, by using Boutet de Monvel calculus (see Boutet de Monvel [5], Rempel-Schulze [20], Schrohe [21]). Our proof of Theorem 2.3 is based on Theorem 4.1. However, in the subelliptic case we cannot use Green's formula in order to characterize the adjoint operator $\mathfrak{A}_{2}^{*}$. Therefore, we shift our attention to the resolvent $\mathfrak{A}_{2}^{*-1}$, instead of $\mathfrak{A}_{2}^{*}$. More precisely, we verify all the conditions of Theorem 4.1 for the resolvent $\left(\mathfrak{A}_{2}^{*}-I\right)^{-1}$ (see Remark 4.2). In the last Section 10 we remark that Theorem 2.3 for the Laplacian $\Delta$ remains valid for a general second-order, uniformly elliptic differential operator $A$ with real smooth coefficients (Theorem 10.1). 


\section{Subelliptic pseudo-differential operators}

Let $\Omega$ be an open subset of $\mathbf{R}^{n}$. A properly supported, pseudo-differential operator $A$ in the Hörmander class $L_{1,0}^{m}(\Omega)$ of order $m \in \mathbf{R}$ is said to be subelliptic with loss of some $\delta \in[0,1)$ if, for every compact $K \subset \Omega, s \in \mathbf{R}$ and $t<s+m-\delta$ there exists a constant $C_{K, s, t}>0$ such that we have the inequality

$$
\|u\|_{H^{s+m-\delta}(\Omega)} \leq C_{K, s, t}\left(\|A u\|_{H^{s}(\Omega)}+\|u\|_{H^{t}(\Omega)}\right) \quad \text { for all } u \in C_{K}^{\infty}(\Omega) .
$$

Here

$$
C_{K}^{\infty}(\Omega):=\text { the space of functions in } C^{\infty}(\Omega) \text { with support in } K .
$$

It is known (see Hörmander [14, Theorem 1.4.3]) that subelliptic operators are hypoelliptic, with loss of $\delta$-derivatives.

Egorov [8] and Hörmander [15] have obtained necessary and sufficient conditions in order that a properly supported, classical pseudo-differential operator $A \in L_{\mathrm{cl}}^{m}(\Omega)$ of order $m$ is subelliptic. More precisely, we have the following theorem (see [15, Theorem 3.4] and [31, Theorem I]):

Theorem 3.1. (Egorov-Hörmander) Let $A$ be a properly supported, pseudodifferential operator in the class $L_{\mathrm{cl}}^{m}(\Omega)$ having the principal symbol $a_{m}(x, \xi)$. Then $A$ is subelliptic with loss of some $\delta \in[0,1)$ if and only if, at every point $x_{0}$ of $\Omega$ there exists a neighborhood $V$ of $x_{0}$ such that the following two conditions are satisfied:

(i) For any point $(x, \xi) \in V \times\left(\mathbf{R}^{n} \backslash\{0\}\right)$, the function

$$
\left(H_{\operatorname{Re} z a_{m}}\right)^{j}\left(\operatorname{Im} z a_{m}\right)(x, \xi)
$$

is different from zero for some complex number $z$ and some non-negative integer $j \leq \delta /(1-\delta)$. Here $H_{f}$ is the Hamilton vector field defined by the formula

$$
H_{f}=\sum_{i=1}^{n} \frac{\partial f}{\partial \xi_{i}} \frac{\partial}{\partial x_{i}}-\sum_{i=1}^{n} \frac{\partial f}{\partial x_{i}} \frac{\partial}{\partial \xi_{i}} .
$$

(ii) If $j$ is an odd integer and is the smallest integer such that the function (3.1) is not identically equal to zero, then the function (3.1) is non-negative for all $(x, \xi) \in V \times\left(\mathbf{R}^{n} \backslash\{0\}\right)$.

\section{Non self-adjoint eigenvalue problems}

Let $A$ be a second-order, uniformly elliptic differential operator with real coefficients on the closure $\bar{\Omega}=\Omega \cup \Gamma$ such that

$$
A=\sum_{i=1}^{n} a^{i j}(x) \frac{\partial^{2}}{\partial x_{i} \partial x_{j}}+\sum_{i=1}^{n} b^{i}(x) \frac{\partial}{\partial x_{i}}+c(x) .
$$


Here:

(1) $a^{i j} \in C^{\infty}(\bar{\Omega})$ and $a^{i j}(x)=a^{j i}(x)$ for all $x \in \bar{\Omega}$ and $1 \leq i, j \leq n$, and there exists a constant $c_{0}>0$ such that

$$
\sum_{i, j=1}^{n} a^{i j}(x) \xi_{i} \xi_{j} \geq c_{0}|\xi|^{2} \quad \text { for all }(x, \xi) \in \bar{\Omega} \times \mathbf{R}^{n}
$$

(2) $b^{i} \in C^{\infty}(\bar{\Omega})$ for all $1 \leq i \leq n$.

(3) $c \in C^{\infty}(\bar{\Omega})$.

We consider a densely defined, closed linear operator

$$
\mathcal{A}: L^{2}(\Omega) \longrightarrow L^{2}(\Omega)
$$

in the Hilbert space $L^{2}(\Omega)$ that satisfies the following four conditions (see Agmon [2, Theorem 15.1]):

(a) The domain $\mathcal{D}(\mathcal{A})$ of definition is a subspace of $H^{2}(\Omega)$ :

$$
\mathcal{D}(\mathcal{A}) \subset H^{2}(\Omega)
$$

(b) $\mathcal{A} u=A u$ for every $u \in \mathcal{D}(\mathcal{A})$.

Its adjoint operator

$$
\mathcal{A}^{*}: L^{2}(\Omega) \longrightarrow L^{2}(\Omega)
$$

is characterized as follows:

(c) The domain $\mathcal{D}\left(\mathcal{A}^{*}\right)$ of definition is a subspace of $H^{2}(\Omega)$ :

$$
\mathcal{D}\left(\mathcal{A}^{*}\right) \subset H^{2}(\Omega)
$$

(d) $\mathcal{A}^{*} u=A^{\prime} u$ for every $u \in \mathcal{D}\left(\mathcal{A}^{*}\right)$.

Here $A^{\prime}$ is the formal adjoint differential operator of $A$.

Then we can prove the following spectral properties of the closed realization $\mathcal{A}$ of $A$ (see [2, Theorems 14.4 and 15.1]):

Theorem 4.1. Assume that the operator $\mathcal{A}$ satisfies the following two conditions:

(A) All rays $\arg \lambda=\theta$ different from the negative axis are rays of minimal growth of the resolvent $(\mathcal{A}-\lambda I)^{-1}$. Namely, there exist constants $R(\theta)>0$ and $C(\theta)>0$, depending on $\theta$, such that we have, for all $|\lambda| \geq R(\theta)$,

$$
\left\|(\mathcal{A}-\lambda I)^{-1}\right\| \leq \frac{C(\theta)}{|\lambda|} .
$$


(B) There exists a positive integer $k$ such that

$$
k>\frac{n+1}{2}
$$

and that

$$
\mathcal{D}\left(\mathcal{A}^{k}\right) \cup \mathcal{D}\left(\left(\mathcal{A}^{*}\right)^{k}\right) \subset H^{2 k}(\Omega) .
$$

Then we have the following four assertions:

(i) The spectrum of $\mathcal{A}$ is discrete and the eigenvalues $\lambda_{j}$ of $\mathcal{A}$ have finite multiplicities.

(ii) The negative axis is a direction of condensation of eigenvalues of $\mathcal{A}$. More precisely, there are only a finite number of eigenvalues outside the angle: $-\pi+\varepsilon<$ $\theta<\pi-\varepsilon$, for each $\varepsilon>0$.

(iii) Let

$$
N(t):=\sum_{\operatorname{Re} \lambda_{j} \geq-t} 1
$$

be the number of eigenvalues $\lambda_{j}$ such that $\operatorname{Re} \lambda_{j} \geq-t$, where each $\lambda_{j}$ is repeated according to its multiplicity. Then the asymptotic eigenvalue distribution formula

$$
N(t)=\frac{1}{(2 \pi)^{n}} \int_{\Omega}|A(x)| d x \cdot t^{n / 2}+o\left(t^{n / 2}\right) \quad \text { as } t \rightarrow+\infty
$$

holds true. Here $|A(x)|$ denotes the volume of the subset

$$
A(x)=\left\{\xi \in \mathbf{R}^{n}: \sum_{i, j=1}^{n} a^{i j}(x) \xi_{i} \xi_{j}<1\right\} .
$$

(iv) The generalized eigenfunctions of $\mathcal{A}$ are complete in the Hilbert space $L^{2}(\Omega)$; they are also complete in the domain $\mathcal{D}(\mathcal{A})$ in the $H^{2}(\Omega)$-norm.

Remark 4.2. Condition (4.1) may be replaced by the following (see Agmon [2, Section 15, p. 263]):

$$
\mathcal{R}\left(\mathcal{A}^{-k}\right) \cup \mathcal{R}\left(\left(\mathcal{A}^{*}\right)^{-k}\right) \subset H^{2 k}(\Omega)
$$

Indeed, it suffices to note that

$$
\begin{aligned}
& \mathcal{R}\left(\mathcal{A}^{-k}\right)=\mathcal{D}\left(\mathcal{A}^{k}\right), \\
& \mathcal{R}\left(\left(\mathcal{A}^{*}\right)^{-k}\right)=\mathcal{D}\left(\left(\mathcal{A}^{*}\right)^{k}\right) .
\end{aligned}
$$




\section{The homogeneous oblique derivative problem}

In this section, by using the Dirichlet problem we consider the homogeneous oblique derivative problem for second-order, uniformly elliptic differential operators in the framework of $L^{p}$ Sobolev spaces.

\subsection{Formulation of the oblique derivative problem}

If $1<p<\infty$, we introduce a maximal domain $H_{A}(\Omega)$ for the operator $\Delta$ in the Banach space $L^{p}(\Omega)$ as follows:

$$
H_{A}(\Omega)=\left\{u \in L^{p}(\Omega): \Delta u \in L^{p}(\Omega)\right\} .
$$

We equip the space $H_{A}(\Omega)$ with the graph norm

$$
\|u\|_{H_{A}(\Omega)}=\left(\|u\|_{L^{p}(\Omega)}^{2}+\|\Delta u\|_{L^{p}(\Omega)}^{2}\right)^{1 / 2} .
$$

The maximal domain $H_{A}(\Omega)$ is a Banach space.

Then we have the following proposition (see [26, Theorem 8.3.2]):

Proposition 5.1. The oblique boundary operator

$$
\begin{aligned}
B \gamma: H_{A}(\Omega) & \longrightarrow B^{-1-1 / p, p}(\Gamma) \\
u & \longmapsto a\left(x^{\prime}\right) \frac{\partial u}{\partial \mathbf{n}}+\alpha\left(x^{\prime}\right) \cdot u
\end{aligned}
$$

is continuous.

Now we can formulate the homogeneous oblique derivative problem as follows: Given a function $f \in L^{p}(\Omega)$, find a function $u \in L^{p}(\Omega)$ such that

$$
\begin{cases}\Delta u=f & \text { in } \Omega, \\ B \gamma u=0 & \text { on } \Gamma .\end{cases}
$$

\subsection{The standard reduction to the boundary}

Let $1<p<\infty$ and $s>-1+1 / p$. Given a function $f \in W^{s, p}(\Omega)$, assume that a function $u \in W^{\sigma, p}(\Omega)$ with $\sigma \leq s+2$ is a solution of problem (2.3). Then, by using Proposition 5.1 we can reduce the study of problem (2.3) to that of a pseudodifferential equation on the boundary $\Gamma$, just as in the classical Fredholm integral equation. In fact, we can prove the following proposition (see [26, Theorem 8.3.3]): 
Proposition 5.2. Let $1<p<\infty, s>-2+1 / p$ and $\sigma \leq s+2$. For a given function $f \in W^{s, p}(\Omega)$, there exists a solution $u \in W^{\sigma, p}(\Omega)$ of problem (2.3) if and only if there exists a solution $\varphi \in B^{\sigma-1 / p, p}(\Gamma)$ of the equation

$$
B \gamma(P \varphi)=-B \gamma(N *(E f)) \quad \text { on } \Gamma
$$

Here $N$ is the Newtonian volume potential and $P$ is the Poisson kernel for the Laplacian, and

$$
E: W^{m, p}(\Omega) \longrightarrow W^{m, p}\left(\mathbf{R}^{n}\right)
$$

is Seeley's extension operator for every non-negative integer $m$ ([1, Theorems 5.21 and 5.22]). Moreover, the solutions $u$ and $\varphi$ are related as follows:

$$
u=\left.(N *(E f))\right|_{\Omega}+P \varphi
$$

If we let

$$
\begin{aligned}
T: C^{\infty}(\Gamma) & \longrightarrow C^{\infty}(\Gamma) \\
\varphi & \longmapsto B \gamma(P \varphi),
\end{aligned}
$$

then we have the formula

$$
T=a\left(x^{\prime}\right) \Pi+\alpha\left(x^{\prime}\right)
$$

where $\Pi$ is the Dirichlet-Neumann operator defined as follows:

$$
\Pi \varphi=\left.\frac{\partial}{\partial \mathbf{n}}(P \varphi)\right|_{\Gamma} \quad \text { for all } \varphi \in C^{\infty}(\Gamma) .
$$

It is well known (cf. [6], [13], [20], [22] and [30]) that the operator $\Pi$ is a classical, elliptic pseudo-differential operator of first order on the boundary $\Gamma$.

More precisely, we can write down the complete symbol $p\left(x^{\prime}, \xi^{\prime}\right)$ of $\Pi$ as follows (cf. [10] and [24, Lemme 2.2]):

$$
\begin{aligned}
p\left(x^{\prime}, \xi^{\prime}\right)= & \left|\xi^{\prime}\right|+\frac{1}{2}\left(\frac{\omega_{x^{\prime}}\left(\widehat{\xi}^{\prime}, \widehat{\xi}^{\prime}\right)}{\left|\xi^{\prime}\right|^{2}}-(n-1) M\left(x^{\prime}\right)\right)-\sqrt{-1} \frac{1}{2} \operatorname{div} \delta_{\left(\xi^{\prime}\right)}\left(x^{\prime}\right) \\
& + \text { terms of order } \leq-1
\end{aligned}
$$

Here:

(a) $\left|\xi^{\prime}\right|$ is the length of $\xi^{\prime}$ with respect to the Riemannian metric of $\Gamma$ induced by the natural metric of $\mathbf{R}^{n}$.

(b) $M\left(x^{\prime}\right)$ is the mean curvature of the boundary $\Gamma$ at $x^{\prime}$. 
(c) $\omega_{x^{\prime}}\left(\widehat{\xi^{\prime}}, \widehat{\xi^{\prime}}\right)$ is the second fundamental form of $\Gamma$ at $x^{\prime}$, while $\widehat{\xi^{\prime}} \in T_{x^{\prime}}(\Gamma)$ is the tangent vector corresponding to the cotangent vector $\xi^{\prime} \in T_{x^{\prime}}^{*}(\Gamma)$ by the duality between $T_{x^{\prime}}(\Gamma)$ and $T_{x^{\prime}}^{*}(\Gamma)$ with respect to the Riemannian metric $\left(g_{i j}\left(x^{\prime}\right)\right)$ of $\Gamma$.

(d) $\operatorname{div} \delta_{\left(\xi^{\prime}\right)}$ is the divergence of a real smooth vector field $\delta_{\left(\xi^{\prime}\right)}$ on $\Gamma$ defined (in local coordinates) by the formula

$$
\delta_{\left(\xi^{\prime}\right)}=\sum_{j=1}^{n-1} \frac{\partial\left|\xi^{\prime}\right|}{\partial \xi_{j}} \frac{\partial}{\partial x_{j}} \quad \text { for } \xi^{\prime} \neq 0
$$

that is,

$$
\operatorname{div} \delta_{\left(\xi^{\prime}\right)}=\frac{1}{\sqrt{\operatorname{det}\left(g_{i j}\left(x^{\prime}\right)\right)}} \sum_{j=1}^{n-1} \frac{\partial}{\partial x_{j}}\left(\sqrt{\operatorname{det}\left(g_{i j}\left(x^{\prime}\right)\right)} \frac{\partial\left|\xi^{\prime}\right|}{\partial \xi_{j}}\right) \quad \text { for } \xi^{\prime} \neq 0
$$

Hence, we find from formula (5.3) that the principal symbol $t_{1}\left(x^{\prime}, \xi^{\prime}\right)$ of the pseudo-differential operator $T$, defined by formula (5.2), is equal to the following:

$$
t_{1}\left(x^{\prime}, \xi^{\prime}\right)=a\left(x^{\prime}\right)\left|\xi^{\prime}\right|+\sqrt{-1}\left[\sum_{k=1}^{n-1} \alpha^{k}\left(x^{\prime}\right) \xi_{k}\right] .
$$

By virtue of Proposition 5.2, we can reduce problem (2.3) to the study of the pseudo-differential operator $T$ on the boundary $\Gamma$. We shall formulate this fact more precisely in terms of functional analysis (cf. [28, Chapter 6]).

First, we associate with the homogeneous problem (2.3) a densely defined, closed linear operator

$$
\mathfrak{A}_{p}: L^{p}(\Omega) \longrightarrow L^{p}(\Omega)
$$

in the Banach space $L^{p}(\Omega)$ as follows.

(a) The domain $\mathcal{D}\left(\mathfrak{A}_{p}\right)$ of definition is the space

$$
\begin{aligned}
\mathcal{D}\left(\mathfrak{A}_{p}\right) & =\left\{u \in H_{A}(\Omega): B \gamma u=0 \text { on } \Gamma\right\} \\
& =\left\{u \in W^{2, p}(\Omega): B \gamma u=0 \text { on } \Gamma\right\} .
\end{aligned}
$$

(b) $\mathfrak{A}_{p} u=\Delta u$ for every $u \in \mathcal{D}\left(\mathfrak{A}_{p}\right)$.

The closedness of $\mathfrak{A}_{p}$ and the regularity property (5.5b) will be proved in Section 8 (see Claim 8.1).

Secondly, by using Proposition 5.2 with $s:=0$ and $\sigma:=2$ we associate with (5.1) a densely defined, closed linear operator

$$
\mathcal{T}_{p}: B^{2-1 / p, p}(\Gamma) \longrightarrow B^{2-1 / p, p}(\Gamma)
$$

in the Banach space $B^{2-1 / p, p}(\Gamma)$ as follows. 
( $\alpha$ ) The domain $\mathcal{D}\left(\mathcal{T}_{p}\right)$ of definition is the space

$$
\mathcal{D}\left(\mathcal{T}_{p}\right)=\left\{\varphi \in B^{2-1 / p, p}(\Gamma): T \varphi \in B^{2-1 / p, p}(\Gamma)\right\} .
$$

( $\beta) \mathcal{T}_{p} \varphi=T \varphi=B \gamma(P \varphi)$ for every $\varphi \in \mathcal{D}\left(\mathcal{T}_{p}\right)$.

Then we obtain the following formula for the indices of the operators $\mathfrak{A}_{p}$ and $\mathcal{T}_{p}$ (see [28, Theorem 6.11]):

Theorem 5.3. Let $1<p<\infty$. If the operator $\mathcal{T}_{p}$ is a Fredholm operator, then the operator $\mathfrak{A}_{p}$ is a Fredholm operator. In this case, we have the formula

$$
\text { ind } \mathfrak{A}_{p}=\operatorname{ind} \mathcal{T}_{p} \text {. }
$$

Moreover, we can prove the following ([29, Proposition 6.8]):

Proposition 5.4. Assume that hypothesis $(\mathrm{H})$ is satisfied. Then the pseudodifferential operators $T$ and $T^{\prime}$ are both subelliptic with loss of some $\delta \in[2 k /(2 k+$ 1), 1) on $\Gamma$. Here $T^{\prime}$ is the transpose of $T$.

By Smith [23] and Guan-Sawyer [11], we find that if condition (H) is satisfied, then the pseudo-differential operator $T$ has a parametrix (see Remark 2.2).

Summing up, we have proved the following ([29, Proposition 6.9]):

Proposition 5.5. If the condition $(\mathrm{H})$ is satisfied, then the operator $\mathcal{T}_{p}$, defined by formula (5.6), is a Fredholm operator for all $1<p<\infty$.

\subsection{Index of the operator $\mathfrak{A}_{p}$}

The next theorem is an immediate consequence of Proposition 5.4 (see [23, Theorem 4.5] and [11, Proposition 5.38]):

Theorem 5.6. Let $1<p<\infty$. Assume that condition $(\mathrm{H})$ is satisfied. Then we have the following two assertions:

(i) If $\varphi \in \mathcal{D}^{\prime}(\Gamma), T \varphi \in B^{\sigma, p}(\Gamma)$ for $\sigma \in \mathbf{R}$, then it follows that $\varphi \in B^{\sigma, p}(\Gamma)$. In particular, we have the assertion

$$
\mathcal{N}\left(\mathcal{T}_{p}\right) \subset C^{\infty}(\Gamma)
$$

(ii) If $\psi \in \mathcal{D}^{\prime}(\Gamma), T^{\prime} \psi \in B^{\sigma, p^{\prime}}(\Gamma)$ for $\sigma \in \mathbf{R}$, then it follows that $\psi \in B^{\sigma, p^{\prime}}(\Gamma)$. In particular, we have the assertion

$$
\mathcal{N}\left(\left(\mathcal{T}_{p}\right)^{\prime}\right) \subset C^{\infty}(\Gamma)
$$


Here the transpose operator $\left(\mathcal{T}_{p}\right)^{\prime}$ of $\mathcal{T}_{p}$ is a densely defined, closed linear operator

$$
\left(\mathcal{T}_{p}\right)^{\prime}: B^{-2+1 / p, p^{\prime}}(\Gamma) \longrightarrow B^{-2+1 / p, p^{\prime}}(\Gamma), \quad p^{\prime}=\frac{p}{p-1}
$$

such that

$$
\left\langle\mathcal{T}_{p} \varphi, \psi\right\rangle=\left\langle\varphi,\left(\mathcal{T}_{p}\right)^{\prime} \psi\right\rangle \quad \text { for all } \varphi \in \mathcal{D}\left(\mathcal{T}_{p}\right) \text { and } \psi \in \mathcal{D}\left(\left(\mathcal{T}_{p}\right)^{\prime}\right),
$$

where $\langle\cdot, \cdot\rangle$ is the duality between the spaces $B^{2-1 / p, p}(\Gamma)$ and $B^{-2+1 / p, p^{\prime}}(\Gamma)$.

Finally, by combining Proposition 5.5, Theorems 5.3 and 5.6 we obtain the following fundamental result of the indices of the operators $\mathfrak{A}_{p}$ ([29, Theorem 6.11]):

Theorem 5.7. If condition $(\mathrm{H})$ is satisfied, then the index ind $\mathfrak{A}_{p}=\operatorname{ind} \mathcal{T}_{p}$ is independent of $p$ for all $1<p<\infty$.

Remark 5.8. By using Agmon's method, we can prove that the index ind $\mathfrak{A}_{p}$ is equal to zero for all $1<p<\infty$ (see [29, Theorem 12.1]).

\section{Uniqueness theorem for the oblique derivative problem}

In this section we consider the following non-homogeneous oblique derivative problem: Given a function $f(x)$ defined in $\Omega$ and a function $\varphi\left(x^{\prime}\right)$ defined on $\Gamma$, find a function $u(x)$ in $\Omega$ such that

$$
\begin{cases}(1-\Delta) u=f & \text { in } \Omega, \\ B \gamma u=a\left(x^{\prime}\right) \frac{\partial u}{\partial \mathbf{n}}+\alpha\left(x^{\prime}\right) \cdot u=\varphi & \text { on } \Gamma .\end{cases}
$$

The purpose of this section is to prove the following uniqueness theorem for the oblique derivative problem (2.4) in the framework of $L^{p}$ Sobolev spaces (see [32, Theorem 2.4] and [11, Remark (b)]):

Theorem 6.1. Assume that the function $a\left(x^{\prime}\right)$ does not change sign on the boundary $\Gamma$ and further that the following hypothesis $(\mathrm{R})$ is satisfied:

$(\mathrm{R})$ The vector field $\alpha\left(x^{\prime}\right)$ is non-zero on the set $\Gamma_{0}=\left\{x^{\prime} \in \Gamma: a\left(x^{\prime}\right)=0\right\}$ of tangency and any integral curve $x\left(t, x_{0}^{\prime}\right)$ of $\alpha$ passing through $x_{0}^{\prime} \in \Gamma_{0}$ at $t=0$ does not lie in the set $\Gamma_{0}$ for an infinite interval of time.

Then every solution $u \in W^{2, p}(\Omega), p>n$, of the problem

$$
\begin{cases}(1-\Delta) u=0 & \text { in } \Omega, \\ B \gamma u=0 & \text { on } \Gamma\end{cases}
$$

is identically equal to zero in $\Omega$. 
Proof. First, it follows from an application of Sobolev's imbedding theorem ([1, Theorem 5.4]) that if $p>n$, we have the continuous injection

$$
W^{2, p}(\Omega) \subset C^{\nu}(\bar{\Omega}) \text { for } 0<\nu \leq 2-\frac{n}{p} .
$$

In particular, we find that every function $u \in W^{2, p}(\Omega)$ with $p>n$ belongs to the space $C^{1}(\bar{\Omega})$ :

$$
u \in C^{1}(\bar{\Omega}),
$$

since $2-n / p>1$ for $n<p<\infty$. Moreover, we may assume that

$$
a\left(x^{\prime}\right) \geq 0 \quad \text { on } \Gamma \text {. }
$$

Our proof of Theorem 6.1 is divided into two steps.

Step 1: The case where $u(x)$ is a constant in $\Omega$. Then we have, by the (6.1),

$$
0=(1-\Delta) u=u \quad \text { in } \Omega \text {. }
$$

Step 2: The case where $u(x)$ is not constant in $\Omega$. In this case, (if necessary replacing by $u$ by $-u$ ) we may assume that there exists a point $x_{0}^{\prime} \in \Gamma$ such that

$$
u\left(x_{0}^{\prime}\right)=\max _{x \in \bar{\Omega}} u(x)>0 .
$$

Then, by applying Hopf's boundary point lemma we obtain that

$$
\frac{\partial u}{\partial \mathbf{n}}\left(x_{0}^{\prime}\right)>0 .
$$

Hence we have, by the boundary condition (6.2),

$$
0=B \gamma u\left(x_{0}^{\prime}\right)=a\left(x_{0}^{\prime}\right) \frac{\partial u}{\partial \mathbf{n}}\left(x_{0}^{\prime}\right)+\alpha\left(x_{0}^{\prime}\right) \cdot u\left(x_{0}^{\prime}\right)=a\left(x_{0}^{\prime}\right) \frac{\partial u}{\partial \mathbf{n}}\left(x_{0}^{\prime}\right),
$$

and so

$$
a\left(x_{0}^{\prime}\right)=0
$$

This implies that

$$
x_{0}^{\prime} \in \Gamma_{0}=\left\{x^{\prime} \in \Gamma: a\left(x^{\prime}\right)=0\right\} .
$$

Let

$$
x\left(t, x_{0}^{\prime}\right)=\left(x^{1}\left(t, x_{0}^{\prime}\right), \ldots, x^{n-1}\left(t, x_{0}^{\prime}\right)\right)
$$

be a maximal integral curve of $\alpha\left(x^{\prime}\right)$ passing through $x_{0}^{\prime}$ at $t=0$. By hypothesis $(\mathrm{R})$, we can find a time $t^{-} \leq 0$ such that

$$
t^{-}=\inf \left\{t<0: a\left(x\left(s, x_{0}^{\prime}\right)\right)=0 \text { for all } s \in[t, 0]\right\} .
$$

Here we remark that the flow exists for all times. 
Then we have, by the fundamental theorem of calculus and the boundary condition (6.2),

$$
\begin{aligned}
u\left(x_{0}^{\prime}\right)-u\left(x\left(t^{-}, x_{0}^{\prime}\right)\right) & =u\left(x\left(0, x_{0}^{\prime}\right)\right)-u\left(x\left(t^{-}, x_{0}^{\prime}\right)\right) \\
& =\int_{t^{-}}^{0} \frac{d}{d s}\left(u\left(x\left(s, x_{0}^{\prime}\right)\right)\right) d s \\
& =\int_{t^{-}}^{0} \sum_{k=1}^{n-1} \frac{\partial u}{\partial x_{k}} \frac{d x_{k}}{d s} d s=\int_{t^{-}}^{0} \sum_{k=1}^{n-1} \alpha_{k} \frac{\partial u}{\partial x_{k}} d s \\
& =\int_{t^{-}}^{0} \alpha\left(x\left(s, x_{0}^{\prime}\right)\right) \cdot u\left(x\left(s, x_{0}^{\prime}\right)\right) d s \\
& =-\int_{t^{-}}^{0} a\left(x\left(s, x_{0}^{\prime}\right)\right) \frac{\partial u}{\partial \mathbf{n}}\left(x\left(s, x_{0}^{\prime}\right)\right) d s .
\end{aligned}
$$

However, by the definition (6.3) of $t^{-}$it follows that

$$
u\left(x_{0}^{\prime}\right)-u\left(x\left(t^{-}, x_{0}^{\prime}\right)\right)=-\int_{0}^{t^{-}} a\left(x\left(s, x_{0}^{\prime}\right)\right) \frac{\partial u}{\partial \mathbf{n}}\left(x\left(s, x_{0}^{\prime}\right)\right) d s=0,
$$

so that

$$
u\left(x\left(t^{-}, x_{0}^{\prime}\right)\right)=u\left(x_{0}^{\prime}\right)=\max _{x \in \bar{\Omega}} u(x)>0 .
$$

Hence, by applying again Hopf's boundary point lemma we obtain that

$$
\frac{\partial u}{\partial \mathbf{n}}\left(x\left(t^{-}, x_{0}^{\prime}\right)\right)>0 .
$$

We choose a number $\varepsilon_{0}>0$ so small that we have, for all $s \in\left[t^{-}-\varepsilon_{0}, t^{-}\right]$,

$$
\begin{aligned}
& \frac{\partial u}{\partial \mathbf{n}}\left(x\left(s, x_{0}^{\prime}\right)\right)>0, \\
& a\left(x\left(s, x_{0}^{\prime}\right)\right) \geq 0 .
\end{aligned}
$$

Then we have, by the fundamental theorem of calculus and the boundary condition (6.2),

$$
\begin{aligned}
u\left(x\left(t^{-}, x_{0}^{\prime}\right)\right)-u\left(x\left(t^{-}-\varepsilon_{0}, x_{0}^{\prime}\right)\right) & =\int_{t^{-}-\varepsilon_{0}}^{t^{-}} \alpha\left(x\left(s, x_{0}^{\prime}\right)\right) \cdot u\left(x\left(s, x_{0}^{\prime}\right)\right) d s \\
& =-\int_{t^{-}-\varepsilon_{0}}^{t^{-}} a\left(x\left(s, x_{0}^{\prime}\right)\right) \frac{\partial u}{\partial \mathbf{n}}\left(x\left(s, x_{0}^{\prime}\right)\right) d s \\
& <0 .
\end{aligned}
$$


However, by formula (6.4) it follows that

$$
u\left(x\left(t^{-}-\varepsilon_{0}, x_{0}^{\prime}\right)\right)>u\left(x\left(t^{-}, x_{0}^{\prime}\right)\right)=u\left(x_{0}^{\prime}\right)=\max _{x \in \bar{\Omega}} u(x) .
$$

This is a contradiction.

The proof of Theorem 6.1 is now complete.

Remark 6.2. The hypothesis ( $\mathrm{R}$ ) is introduced by Winzell [32] (see MelinSjöstrand [17] and [18]). By definition, the hypothesis (R) implies that every integral curve $x\left(t, x_{0}^{\prime}\right)$ of $\alpha$ passing through $x_{0}^{\prime} \in \Gamma_{0}$ at $t=0$ goes out of the set $\Gamma_{0}$ of tangency in a finite time. On the other hand, the hypothesis $(\mathrm{H})$ implies that every integral curve $x\left(t, x_{0}^{\prime}\right)$ goes out of $\Gamma_{0}$ instantaneously.

\section{A special reduction to the boundary}

In this section, by using the Poisson kernel $P_{2}^{\text {ext }}$ of $1-\Delta$ in the exterior domain $\bar{\Omega}^{c}=\mathbf{R}^{n} \backslash \bar{\Omega}$ we reduce the homogeneous oblique derivative problem (2.5) to the study of a first-order, pseudo-differential operator $T$ on the boundary $\Gamma$ (Proposition 7.1), just as in Smith [23] and Guan-Sawyer [11].

Step 1: Let $f \in W^{s, p}(\Omega)$ with $1<p<\infty$ and $s>-1+1 / p$. We denote by $f_{0}$ the extension of $f$ to Euclidean space $\mathbf{R}^{n}$ with $f_{0} \equiv 0$ outside $\Omega$ :

$$
f_{0}(x)= \begin{cases}f(x) & \text { for } x \in \Omega, \\ 0 & \text { for } x \in \mathbf{R}^{n} \backslash \Omega .\end{cases}
$$

Let $G_{2}(x)$ be the Bessel potential of order 2, that is,

$$
\widehat{G}_{2}(\xi)=\int_{\mathbf{R}^{n}} e^{-i x \xi} G_{2}(x) d x=\frac{1}{1+|\xi|^{2}} .
$$

If we let

$$
\begin{aligned}
r^{+} G_{2} f(x) & :=G_{2} * f_{0}(x)=\int_{\mathbf{R}^{n}} G_{2}(x-y) f_{0}(y) d y \\
& =\int_{\Omega} G_{2}(x-y) f(y) d y \quad \text { for all } x \in \Omega
\end{aligned}
$$

then we obtain from the transmission property of the Bessel potential $G_{2}(x)$ (see Boutet de Monvel [5], Rempel-Schulze [20, p. 161, Theorem 2]) that

$$
r^{+} G_{2} f \in W^{s+2, p}(\Omega) \text { for } s>-1+1 / p,
$$


and further ([20, p. 100, Lemma 5]) that

$$
(1-\Delta) r^{+} G_{2} f=f \quad \text { in } \Omega .
$$

On the other hand, by the trace theorem $([1$, Theorem 7.39$])$ it follows that

$$
B \gamma\left(G_{2} * f_{0}\right)=B \gamma\left(r^{+} G_{2} f\right) \in B^{s+1-1 / p, p}(\Gamma) \text { for } s>-1+1 / p .
$$

If a function $u \in L^{p}(\Omega)$ satisfies the equation

$$
\begin{cases}(1-\Delta) u=f & \text { in } \Omega, \\ B \gamma u=0 & \text { on } \Gamma\end{cases}
$$

then it follows that

$$
(1-\Delta)\left(r^{+} G_{2} f-u\right)=f-f=0 \quad \text { in } \Omega
$$

We let

$$
\psi=\left.\left(r^{+} G_{2} f-u\right)\right|_{\Gamma}
$$

If $P_{2}$ is the Poisson kernel of the elliptic differential operator $1-\Delta$ in $\Omega$, then we have the formula

$$
r^{+} G_{2} f-u=P_{2} \psi \quad \text { in } \Omega
$$

or equivalently,

$$
u=r^{+} G_{2} f-P_{2} \psi \text { in } \Omega .
$$

Then we find that the boundary condition

$$
B \gamma u=\frac{\partial u}{\partial \boldsymbol{\nu}}=\alpha\left(x^{\prime}\right) \cdot u+\left.a\left(x^{\prime}\right) \frac{\partial u}{\partial \mathbf{n}}\right|_{\Gamma}=0 \quad \text { on } \Gamma
$$

is equivalent to the following condition:

$$
\begin{aligned}
0 & =B \gamma\left(r^{+} G_{2} f\right)-B \gamma\left(P_{2} \psi\right) \\
& =B \gamma\left(G_{2} * f_{0}\right)-\left.\alpha\left(x^{\prime}\right) \cdot\left(P_{2} \psi\right)\right|_{\Gamma}-\left.a\left(x^{\prime}\right) \frac{\partial}{\partial \mathbf{n}}\left(P_{2} \psi\right)\right|_{\Gamma} \\
& =B \gamma\left(G_{2} * f_{0}\right)-\left(\alpha\left(x^{\prime}\right) \cdot \psi+\left.a\left(x^{\prime}\right) \frac{\partial}{\partial \mathbf{n}}\left(P_{2} \psi\right)\right|_{\Gamma}\right) \text { on } \Gamma
\end{aligned}
$$

If we let

$$
\begin{aligned}
T_{2}: C^{\infty}(\Gamma) & \longrightarrow C^{\infty}(\Gamma) \\
\varphi & \longmapsto B \gamma\left(P_{2} \varphi\right),
\end{aligned}
$$


then we have the formula

$$
T_{2}=a\left(x^{\prime}\right) \Pi_{2}+\alpha\left(x^{\prime}\right)
$$

where $\Pi_{2}$ is the Dirichlet-Neumann operator defined as follows:

$$
\Pi_{2} \varphi:=\left.\frac{\partial}{\partial \mathbf{n}}\left(P_{2} \varphi\right)\right|_{\Gamma} \text { for all } \varphi \in C^{\infty}(\Gamma) .
$$

Therefore, we obtain from formulas (7.1) and (7.2) that

$$
T_{2} \psi=\alpha\left(x^{\prime}\right) \cdot \psi+a\left(x^{\prime}\right) \Pi_{2} \psi=B \gamma\left(G_{2} * f_{0}\right) \quad \text { on } \Gamma .
$$

This is a generalization of the classical Fredholm integral equation.

In view of [24, Lemme 2.2], we find that the complete symbol $p_{2}\left(x^{\prime}, \xi^{\prime}\right)$ of $\Pi_{2}$ is given by the formula (cf. formula (5.3))

$$
\begin{aligned}
p_{2}\left(x^{\prime}, \xi^{\prime}\right)= & \sqrt{\left|\xi^{\prime}\right|^{2}+1}+\frac{1}{2}\left(\frac{\omega_{x^{\prime}}\left(\widehat{\xi}^{\prime}, \widehat{\xi}^{\prime}\right)}{\left|\xi^{\prime}\right|^{2}+1}-(n-1) M\left(x^{\prime}\right)\right) \\
& -\frac{\sqrt{-1}}{2} \frac{1}{\sqrt{\operatorname{det}\left(g_{i j}\left(x^{\prime}\right)\right)}} \sum_{j=1}^{n-1} \frac{\partial}{\partial x_{j}}\left(\sqrt{\operatorname{det}\left(g_{i j}\left(x^{\prime}\right)\right)} \frac{\partial}{\partial \xi_{j}}\left(\sqrt{\left|\xi^{\prime}\right|^{2}+1}\right)\right) \\
& + \text { terms of order } \leq-1 .
\end{aligned}
$$

Step 2: On the other hand, since the function $f_{0}$ is compactly supported in $\mathbf{R}^{n}$, it follows that the function

$$
G_{2} * f_{0}(x)=\int_{\Omega} G_{2}(x-y) f(y) d y, \quad x \in \mathbf{R}^{n},
$$

satisfies the homogeneous equation

$$
(1-\Delta) G_{2} * f_{0}=f_{0}=0
$$

in the exterior domain

$$
\bar{\Omega}^{c}=\mathbf{R}^{n} \backslash \bar{\Omega},
$$

and vanishes at infinity.

If $P_{2}^{\text {ext }}$ is the Poisson kernel of the elliptic differential operator $1-\Delta$ in the exterior domain $\bar{\Omega}^{c}$, then we have the formula

$$
G_{2} * f_{0}=P_{2}^{\operatorname{ext}}\left(\left.\left(G_{2} * f_{0}\right)\right|_{\Gamma}\right) \text { in } \bar{\Omega}^{c} .
$$

We recall that the analysis of the Poisson kernel $P_{2}^{\text {ext }}$ can be reduced to that of compact domains by using the Kelvin transform (see [3, Chapter 4]). 
Hence we have, by formula (7.5),

$$
B \gamma\left(G_{2} * f_{0}\right)=B \gamma\left(P_{2}^{\operatorname{ext}}\left(\left.\left(G_{2} * f_{0}\right)\right|_{\Gamma}\right)\right)
$$

However, it should be noticed that the outward normal field $\mathbf{n}$ to $\Gamma$ in the interior domain $\Omega$ is the inward normal for the exterior domain $\bar{\Omega}^{c}$.

Therefore, if we define the Dirichlet-Neumann operator $\Pi_{2}^{\text {ext }}$ by the formula

$$
\Pi_{2}^{\text {ext }} \varphi:=\left.\frac{\partial}{\partial(-\mathbf{n})}\left(P_{2}^{\text {ext }} \varphi\right)\right|_{\Gamma} \text { for all } \widetilde{\varphi} \in C^{\infty}(\Gamma),
$$

then we have the formula

$$
\begin{aligned}
B \gamma\left(G_{2} * f_{0}\right) & =B \gamma\left(P_{2}^{\operatorname{ext}}\left(\left.\left(G_{2} * f_{0}\right)\right|_{\Gamma}\right)\right) \\
& =\alpha\left(x^{\prime}\right) \cdot\left(\left.\left(G_{2} * f_{0}\right)\right|_{\Gamma}\right)+\left.a\left(x^{\prime}\right) \frac{\partial}{\partial \mathbf{n}}\left(P_{2}^{\operatorname{ext}}\left(\left.\left(G_{2} * f_{0}\right)\right|_{\Gamma}\right)\right)\right|_{\Gamma} \\
& =\alpha\left(x^{\prime}\right) \cdot\left(\left.\left(G_{2} * f_{0}\right)\right|_{\Gamma}\right)-\left.a\left(x^{\prime}\right) \frac{\partial}{\partial(-\mathbf{n})}\left(P_{2}^{\operatorname{ext}}(\theta)\left(\left.\left(G_{2} * f_{0}\right)\right|_{\Gamma}\right)\right)\right|_{\Gamma} \\
& =\left(\alpha\left(x^{\prime}\right)-a\left(x^{\prime}\right) \Pi_{2}^{\text {ext }}\right)\left(\left.\left(G_{2} * f_{0}\right)\right|_{\Gamma}\right) \text { on } \Gamma
\end{aligned}
$$

In view of formula (7.4), we find that the complete symbol $p_{2}^{\text {ext }}\left(x^{\prime}, \xi^{\prime}\right)$ of $\Pi_{2}^{\text {ext }}$ is given by the formula

$$
\begin{aligned}
p_{2}^{\text {ext }}\left(x^{\prime}, \xi^{\prime}\right)= & \sqrt{\left|\xi^{\prime}\right|^{2}+1}-\frac{1}{2}\left(\frac{\omega_{x^{\prime}}\left(\widehat{\xi}^{\prime}, \widehat{\xi}^{\prime}\right)}{\left|\xi^{\prime}\right|^{2}+1}-(n-1) M\left(x^{\prime}\right)\right) \\
& -\frac{\sqrt{-1}}{2} \frac{1}{\sqrt{\operatorname{det}\left(g_{i j}\left(x^{\prime}\right)\right)}} \sum_{j=1}^{n-1} \frac{\partial}{\partial x_{j}}\left(\sqrt{\operatorname{det}\left(g_{i j}\left(x^{\prime}\right)\right)} \frac{\partial}{\partial \xi_{j}}\left(\sqrt{\left|\xi^{\prime}\right|^{2}+1}\right)\right) \\
& + \text { terms of order } \leq-1 .
\end{aligned}
$$

Step 3: By combining formulas (7.3) and (7.6), we have proved the following fundamental proposition (cf. Proposition 5.2):

Proposition 7.1. Let $f \in W^{s, p}(\Omega)$ with $1<p<\infty$ and $s>-1+1 / p$. Then the homogeneous oblique derivative problem

$$
\begin{cases}(1-\Delta) u=f & \text { in } \Omega \\ B \gamma u=a\left(x^{\prime}\right) \frac{\partial u}{\partial \mathbf{n}}+\alpha\left(x^{\prime}\right) \cdot u=0 & \text { on } \Gamma\end{cases}
$$

can be reduced to the study of the pseudo-differential equation

$$
T_{2} \psi=\left(\alpha\left(x^{\prime}\right)+a\left(x^{\prime}\right) \Pi_{2}\right) \psi=\left(\alpha\left(x^{\prime}\right)-a\left(x^{\prime}\right) \Pi_{2}^{\text {ext }}\right)\left(\left.\left(G_{2} * f_{0}\right)\right|_{\Gamma}\right) \quad \text { on } \Gamma
$$


where

$$
\psi=\left.\left(r^{+} G_{2} f-u\right)\right|_{\Gamma},
$$

and

$$
\begin{aligned}
& \left.\left(G_{2} * f_{0}\right)\right|_{\Omega}=r^{+} G_{2} f \in W^{s+2, p}(\Omega), \\
& \left.\left(G_{2} * f_{0}\right)\right|_{\Gamma}=\left.r^{+} G_{2} f\right|_{\Gamma}=\gamma_{0}\left(r^{+} G_{2} f\right) \in B^{s+2-1 / p, p}(\Gamma) .
\end{aligned}
$$

Remark 7.2. It should be noticed that the pseudo-differential equation (7.8) corresponds to the equation in Smith [23, p. 100]

$$
\left(X_{T}+\alpha\left(x^{\prime}\right) Q\right) v=\left(X_{T}-\alpha\left(x^{\prime}\right) Q^{\mathrm{Ext}}\right)\left(\left.E f_{0}\right|_{\partial \Omega}\right)
$$

with

$$
\begin{array}{ll}
X_{T}:=\alpha, & \alpha\left(x^{\prime}\right):=a\left(x^{\prime}\right), \\
Q:=\Pi_{2}, & Q^{\mathrm{Ext}}:=\Pi_{2}^{\mathrm{ext}}
\end{array}
$$

(see also the boundary equation (2.3) in Guan-Sawyer [11]).

\section{Representation formula of the resolvent}

By combining Proposition 7.1 and Theorem 6.1, we can obtain the following fundamental theorem essentially due to Smith [23, Main Theorem] and GuanSawyer [11, Theorem 2, part (i)]:

Theorem 8.1. Let $1<p<\infty$. Assume that the condition $(\mathrm{H})$ is satisfied. If $f \in W^{s, p}(\Omega)$ with $s>-1+1 / p$, then every solution $u \in L^{p}(\Omega)$ of the homogeneous oblique derivative problem

$$
\begin{cases}(1-\Delta) u=f & \text { in } \Omega, \\ B \gamma u=0 & \text { on } \Gamma\end{cases}
$$

belongs to the space $W^{s+2, p}(\Omega)$. Moreover, every solution $u \in W^{s+2, p}(\Omega)$ can be uniquely expressed in the form

$$
u=r^{+} G_{2} f-P_{2}\left(S_{2}\left(\alpha\left(x^{\prime}\right)-a\left(x^{\prime}\right) \Pi_{2}^{\text {ext }}\right)\left(\left.r^{+} G_{2} f\right|_{\Gamma}\right)\right) .
$$

Here:

(i) $P_{2}: B^{s+2-1 / p, p}(\Gamma) \rightarrow W^{s+2, p}(\Omega)$ is the Poisson kernel for the elliptic differential operator $1-\Delta$.

(ii) $S_{2}$ is the inverse of the pseudo-differential operator $T_{2}=B P_{2}$ in the Banach space $L^{p}(\Gamma)$. 
Proof. (1) First, we find from Proposition 7.1 and Theorem 5.6 that every solution $u \in L^{p}(\Omega)$ of problem $(2.5)$ with $f:=0$ belongs to the space $C^{\infty}(\bar{\Omega})$. Hence the uniqueness theorem for problem (2.5) follows from an application of Theorem 6.1 and Remark 6.2.

(2) Therefore, we obtain from the proof of Proposition 7.1 that every solution $u \in W^{s+2, p}(\Omega)$ can be uniquely expressed by formula (8.1). It should be noticed that the boundary operator

$$
R:=S_{2}\left(\alpha\left(x^{\prime}\right)-a\left(x^{\prime}\right) \Pi_{2}^{\mathrm{ext}}\right)
$$

is bounded on the Besov space $B^{s+2-1 / p, p}(\Gamma)$. In other words, $\psi$ gains 0-derivatives from $\left.\left(G_{2} * f_{0}\right)\right|_{\Gamma}$ in (7.8). Indeed, we have the following four assertions:

(a) By formula (7.4), it follows that the symbol of the inverse $S_{2}$ of $T_{2}=B P_{2}=$ $\alpha\left(x^{\prime}\right) \cdot+a\left(x^{\prime}\right) \Pi_{2}$ is in the symbol class $E S_{\rho}^{-1}$ (see [23, Definition 3.11]).

(b) By formula (7.7), it follows that the symbol of $\alpha\left(x^{\prime}\right)-a\left(x^{\prime}\right) \Pi_{2}^{\text {ext }}$ is in the symbol class $E S_{\rho}^{1}([23$, Theorem 3.11]).

(c) By applying [23, Theorem 3.14] to our situation, we find that the symbol of the product $S_{2}\left(\alpha\left(x^{\prime}\right)-a\left(x^{\prime}\right) \Pi_{2}^{\text {ext }}\right)$ is in the symbol class $E S_{\rho}^{0}$.

(d) The operator $R=S_{2}\left(\alpha\left(x^{\prime}\right)-a\left(x^{\prime}\right) \Pi_{2}^{\text {ext }}\right)$ is bounded on the Besov space $B^{\sigma, p}(\Gamma)$ for every $\sigma \in \mathbf{R}([23$, Theorem 3.13] and [11, Section 6$])$.

The proof of Theorem 8.1 is complete.

In this way, by using the representation formula (8.1) of the solution $u$ we can obtain the following regularity property for the resolvent $\left(I-\mathfrak{A}_{p}\right)^{-1}$ :

Corollary 8.2. Let $1<p<\infty$. Assume that the condition $(\mathrm{H})$ is satisfied. Then the resolvent $\left(I-\mathfrak{A}_{p}\right)^{-1}$, given by the formula

$$
\left(I-\mathfrak{A}_{p}\right)^{-1} f=r^{+} G_{2} f-P_{2}\left(S_{2}\left(\alpha\left(x^{\prime}\right)-a\left(x^{\prime}\right) \Pi_{2}^{\text {ext }}\right)\left(\left.r^{+} G_{2} f\right|_{\Gamma}\right)\right),
$$

maps $W^{s, p}(\Omega)$ continuously into $W^{s+2, p}(\Omega)$ for every $s>-1+1 / p$.

Indeed, it suffices to note the following facts:

$$
r^{+} G_{2} f \in W^{s+2, p}(\Omega),
$$

and

$$
\begin{aligned}
& \left.r^{+} G_{2} f\right|_{\Gamma}=\gamma_{0}\left(r^{+} G_{2} f\right) \in B^{s+2-1 / p, p}(\Gamma), \\
& S_{2}\left(\alpha\left(x^{\prime}\right)-a\left(x^{\prime}\right) \Pi_{2}^{\mathrm{ext}}\right)\left(\left.r^{+} G_{2} f\right|_{\Gamma}\right) \in B^{s+2-1 / p, p}(\Gamma), \\
& P_{2}: B^{s+2-1 / p, p}(\Gamma) \longrightarrow W^{s+2, p}(\Omega) .
\end{aligned}
$$

Now we are in a position to prove the closedness of $\mathfrak{A}_{p}$ and the regularity property $(5.5 b)$ : 
Claim 8.1. The operator $\mathfrak{A}_{p}: L^{p}(\Omega) \rightarrow L^{p}(\Omega)$ is closed for all $1<p<\infty$. Moreover, we have the assertion

$$
\mathcal{D}\left(\mathfrak{A}_{p}\right)=\left\{u \in H_{A}(\Omega): B \gamma u=0 \text { on } \Gamma\right\} \subset W^{2, p}(\Omega) .
$$

This implies the regularity property (5.5b).

Proof. The proof of Claim 8.1 is divided into two steps.

Step 1: Let $\left\{u_{j}\right\}$ be an arbitrary sequence in the domain $\mathcal{D}\left(\mathfrak{A}_{p}\right)$ such that

$$
\begin{cases}u_{j} \longrightarrow u & \text { in } L^{p}(\Omega), \\ \Delta u_{j} \longrightarrow v & \text { in } L^{p}(\Omega) .\end{cases}
$$

Since $\Delta: L^{p}(\Omega) \rightarrow \mathcal{D}^{\prime}(\Omega)$ is continuous, it follows that

$$
\Delta u_{j} \longrightarrow \Delta u \quad \text { in } \mathcal{D}^{\prime}(\Omega),
$$

so that

$$
\Delta u=v \in L^{p}(\Omega)
$$

This proves that

$$
\left\{\begin{array}{l}
u \in H_{A}(\Omega), \\
u_{j} \longrightarrow u
\end{array} \text { in } H_{A}(\Omega) .\right.
$$

Hence we have, by Proposition 5.1,

$$
B \gamma u=\lim _{j \rightarrow \infty} B \gamma u_{j}=0 \quad \text { in } B^{-1-1 / p, p}(\Gamma) .
$$

By combining assertions (8.4) and (8.5), we obtain that

$$
\left\{\begin{array}{l}
u \in \mathcal{D}\left(\mathfrak{A}_{p}\right), \\
\mathfrak{A}_{p} u=v
\end{array}\right.
$$

This proves the closedness of $\mathfrak{A}_{p}$ defined by formula (5.5a).

Step 2: Moreover, by applying Theorem 8.1 with $s:=0$ we find that

$$
\begin{aligned}
& \left\{\begin{array}{l}
u \in H_{A}(\Omega), \\
B \gamma u=0 \text { on } \Gamma
\end{array}\right. \\
& \Longrightarrow \\
& \left\{\begin{array}{l}
(1-\Delta) u \in L^{p}(\Omega), \\
B \gamma u=0 \text { on } \Gamma
\end{array}\right.
\end{aligned}
$$




$$
\Longrightarrow=W^{2, p}(\Omega) .
$$

This proves the desired assertion (8.3).

The proof of Claim 8.1 is complete.

\section{Proof of Theorem 2.3 via Boutet de Monvel calculus}

In this section we prove Theorem 2.3, by using Boutet de Monvel calculus (see [5], [20] and [27, Appendix B]). Our proof of Theorem 2.3 is based on Theorem 4.1 and Remark 4.2. More precisely, we have only to verify condition (4.2) for the resolvent $\left(I-\mathfrak{A}_{2}\right)^{-1}$ given by formula (8.2) with $p:=2$.

First, by Corollary 8.2 with $p:=2$ it follows that

$$
\mathcal{R}\left(\left(I-\mathfrak{A}_{2}\right)^{-k}\right) \subset H^{2 k}(\Omega) \text { for all positive integer } k .
$$

Secondly, the next theorem with $p:=2$ proves that

$$
\mathcal{R}\left(\left(I-\mathfrak{A}_{2}^{*}\right)^{-k}\right) \subset H^{2 k}(\Omega) \text { for all positive integer } k .
$$

Theorem 9.1. Assume that the condition $(\mathrm{H})$ is satisfied. If $\left(I-\mathfrak{A}_{p}\right)^{-1}$ is the resolvent defined by formula (8.2), then its adjoint operator $\left(\left(I-\mathfrak{A}_{p}\right)^{-1}\right)^{*}=$ $\left(I-\mathfrak{A}_{p}^{*}\right)^{-1}$ maps $W^{s, p}(\Omega)$ continuously into $W^{s+2, p}(\Omega)$ for every $s>-1+1 / p$.

Proof. The proof of Theorem 9.1 is divided into five steps.

Step 1: We recall that the operator $r^{+} G_{2}$ is defined by the formula

$$
r^{+} G_{2} f(x):=G_{2} * f_{0}(x)=\int_{\Omega} G_{2}(x-y) f(y) d y \quad \text { for all } x \in \Omega .
$$

Then the operator

$$
r^{+} G_{2}: W^{s, p}(\Omega) \longrightarrow W^{s+2, p}(\Omega)
$$

is continuous for every $s>-1+1 / p([20$, p. 162, Theorem 2]). More precisely, it is a Green operator of order -2 with symbol ([20, p. 119, Proposition 3])

$$
\frac{1}{|\xi|^{2}+1}=\frac{1}{\left\langle\xi^{\prime}\right\rangle^{2}+\tau^{2}}, \quad \xi=\left(\xi^{\prime}, \tau\right),\left\langle\xi^{\prime}\right\rangle=\sqrt{1+\left|\xi^{\prime}\right|^{2}}
$$

The adjoint $\left(r^{+} G_{2}\right)^{*}$ of $r^{+} G_{2}$ is also a Green operator of order -2 with symbol ([20, p. 102, Proposition 6])

$$
\frac{1}{|\xi|^{2}+1}=\frac{1}{\left\langle\xi^{\prime}\right\rangle^{2}+\tau^{2}}
$$


Hence it follows $([20$, p. 161, Theorem 2]) that the adjoint

$$
\left(r^{+} G_{2}\right)^{*}: W^{s, p}(\Omega) \longrightarrow W^{s+2, p}(\Omega)
$$

is continuous for every $s>-1+1 / p$.

Step 2: By Step 1, we can define a continuous operator

$$
\gamma_{0}\left(r^{+} G_{2}\right): W^{s, p}(\Omega) \longrightarrow B^{s+2-1 / p, p}(\Gamma)
$$

by the formula

$$
\gamma_{0}\left(r^{+} G_{2} f\right)\left(x^{\prime}\right):=G_{2} * f_{0}\left(x^{\prime}\right)=\int_{\Omega} G_{2}\left(x^{\prime}-y\right) f(y) d y \quad \text { for all } x^{\prime} \in \Gamma .
$$

Then it is a trace operator of order -2 with the principal symbol ([20, p. 100, Lemma 4])

$$
\frac{1}{2\left\langle\xi^{\prime}\right\rangle} \cdot \frac{1}{\left\langle\xi^{\prime}\right\rangle-i \tau}
$$

The adjoint $\left(\gamma_{0}\left(r^{+} G_{2}\right)\right)^{*}$ of $\gamma_{0}\left(r^{+} G_{2}\right)$ is a potential operator of order -2 with the principal symbol ([20, p. 102, Proposition 6])

$$
\frac{1}{2\left\langle\xi^{\prime}\right\rangle} \cdot \frac{1}{\left\langle\xi^{\prime}\right\rangle+i \tau} \text {. }
$$

Hence it follows ([20, p. 161, Theorem 2]) that the operator

$$
\left(\gamma_{0}\left(r^{+} G_{2}\right)\right)^{*}: B^{s+1-1 / p, p}(\Gamma) \longrightarrow W^{s+2, p}(\Omega)
$$

is continuous for every $s \in \mathbf{R}$.

Step 3: The Poisson kernel

$$
P_{2}: B^{s+2-1 / p, p}(\Gamma) \longrightarrow W^{s+2, p}(\Omega)
$$

is continuous for every $s \in \mathbf{R}$ ([20, p. 161, Theorem 2]). More precisely, it is a potential operator of order -1 with the principal symbol ([20, p. 125, Proposition 2$])$

$$
\frac{1}{\left\langle\xi^{\prime}\right\rangle+i \tau} \text {. }
$$

The adjoint $P_{2}^{*}$ of $P_{2}$ is a trace operator of order -1 with the principal symbol ([20, p. 102, Proposition 6])

$$
\frac{1}{\left\langle\xi^{\prime}\right\rangle-i \tau}
$$

Hence it follows ([20, p. 162, Theorem 2]) that the adjoint

$$
P_{2}^{*}: W^{s, p}(\Omega) \longrightarrow B^{s+1-1 / p, p}(\Gamma)
$$

is continuous for every $s>-1+1 / p$. 
Step 4: The symbol of the adjoint $R^{*}$ of the pseudo-differential operator

$$
R=S_{2}\left(\alpha\left(x^{\prime}\right)-a\left(x^{\prime}\right) \Pi_{2}^{\text {ext }}\right)
$$

is in the same symbol class $E S_{\rho}^{0}([23$, Theorem 3.15]). Therefore, we obtain $([23$, Theorem 3.13]) that the adjoint $R^{*}$ is also bounded on the Besov space $B^{\sigma, p}(\Gamma)$ for every $\sigma \in \mathbf{R}$. In particular, we have the assertion

$$
R^{*}=\left(S_{2}\left(\alpha\left(x^{\prime}\right)-a\left(x^{\prime}\right) \Pi_{2}^{\mathrm{ext}}\right)\right)^{*}: B^{s+1-1 / p, p}(\Gamma) \longrightarrow B^{s+1-1 / p, p}(\Gamma) .
$$

Step 5: Now we remark that the resolvent $\left(I-\mathfrak{A}_{p}\right)^{-1}$ given by formula (8.2) can be written in the form

$$
\left(I-\mathfrak{A}_{p}\right)^{-1}=r^{+} G_{2}-P_{2} R\left(\gamma_{0}\left(r^{+} G_{2}\right)\right) .
$$

Therefore, we obtain that the adjoint $\left(\left(I-\mathfrak{A}_{p}\right)^{-1}\right)^{*}$ is given by the formula

$$
\left(\left(I-\mathfrak{A}_{p}\right)^{-1}\right)^{*}=\left(r^{+} G_{2}\right)^{*}-\left(\gamma_{0}\left(r^{+} G_{2}\right)\right)^{*} R^{*} P_{2}^{*},
$$

and further from assertions (9.1) through (9.4) that it maps $W^{s, p}(\Omega)$ continuously into $W^{s+2, p}(\Omega)$ for every $s>-1+1 / p$.

The proof of Theorem 9.1 is complete.

Now Theorem 2.3 follows from an application of Theorem 4.1 and Remark 4.2 with $\mathcal{A}:=\mathfrak{A}_{2}-I$.

\section{Concluding remarks}

(I) We replace the Laplacian $\Delta$ by a second-order, uniformly elliptic differential operator $A$ with real smooth coefficients on the closure $\bar{\Omega}=\Omega \cup \Gamma$ (just as in Section 4)

$$
A=\sum_{i=1}^{n} a^{i j}(x) \frac{\partial^{2}}{\partial x_{i} \partial x_{j}}+\sum_{i=1}^{n} b^{i}(x) \frac{\partial}{\partial x_{i}}+c(x),
$$

and consider the homogeneous oblique derivative problem

$$
\begin{cases}(A-\lambda) u=f & \text { in } \Omega, \\ B \gamma u=a\left(x^{\prime}\right) \frac{\partial u}{\partial \mathbf{n}}+\alpha\left(x^{\prime}\right) \cdot u=0 & \text { on } \Gamma .\end{cases}
$$

We introduce a densely defined, closed linear operator

$$
\mathcal{A}: L^{2}(\Omega) \longrightarrow L^{2}(\Omega)
$$

in the Hilbert space $L^{2}(\Omega)$ as follows: 
(a) The domain $\mathcal{D}(\mathcal{A})$ of definition is the space

$$
\mathcal{D}(\mathcal{A})=\left\{u \in H^{2}(\Omega): B \gamma u=0 \text { on } \Gamma\right\} .
$$

(b) $\mathcal{A} u=A u$ for every $u \in \mathcal{D}(\mathcal{A})$.

Then, by combining Agmon [2, Theorems 14.4 and 15.1] with [29, Theorem 2.2] we can generalize Theorem 2.3 as follows (see [29, Corollary 2.3]):

Theorem 10.1. Assume that the hypothesis $(\mathrm{H})$ is satisfied. Then the closed realization $\mathcal{A}$ of $A$ enjoys the following five spectral properties:

(i) The spectrum of $\mathcal{A}$ is discrete and the eigenvalues $\lambda_{j}$ of $\mathcal{A}$ have finite multiplicities.

(ii) All rays $\arg \lambda=\theta$ different from the negative axis are rays of minimal growth of the resolvent $(\mathcal{A}-\lambda I)^{-1}$ :

$$
\left\|(\mathcal{A}-\lambda I)^{-1}\right\| \leq \frac{C(\theta)}{|\lambda|} \quad \text { for all }|\lambda| \geq R(\theta) .
$$

(iii) The negative axis is a direction of condensation of eigenvalues of $\mathcal{A}$; more precisely, for each $\varepsilon>0$ there are only a finite number of eigenvalues outside the angle: $-\pi+\varepsilon<\theta<\pi-\varepsilon$.

(iv) Let

$$
N(t):=\sum_{\operatorname{Re} \lambda_{j} \geq-t} 1
$$

be the number of eigenvalues $\lambda_{j}$ such that $\operatorname{Re} \lambda_{j} \geq-t$, where each $\lambda_{j}$ is repeated according to its multiplicity. Then the asymptotic eigenvalue distribution formula

$$
N(t)=\frac{1}{(2 \pi)^{n}} \int_{\Omega}|A(x)| d x \cdot t^{n / 2}+o\left(t^{n / 2}\right) \quad \text { as } t \rightarrow+\infty
$$

holds true. Here $|A(x)|$ denotes the volume of the subset $A(x)=\left\{\xi \in \mathbf{R}^{n}\right.$ : $\left.\sum_{i, j=1}^{n} a^{i j}(x) \xi_{i} \xi_{j}<1\right\}$.

(v) The generalized eigenfunctions of $\mathcal{A}$ are complete in the Hilbert space $L^{2}(\Omega)$; they are also complete in the domain $\mathcal{D}(\mathcal{A})$ in the $H^{2}(\Omega)$-norm.

Indeed, in view of formula (5.4) it suffices to note that there is a homotopy through elliptic symbols between the two elliptic differential operators $A$ and $\Delta$ if we take

$$
A_{t}:=t A+(1-t) \Delta \text { for } 0 \leq t \leq 1
$$

(II) In the near future, we would like to apply Theorems 2.3 and 10.1 to provide numerical solutions of the linearized fixed gravimetric boundary value problem on the real Earth surface topography in the degenerate (non-coercive) case, generalizing Holota [12] and Čunderlík-Mikula-Mojzeš [7]. 
Acknowledgements. The author is grateful to the referee for many valuable suggestions and comments, which have improved substantially the presentation of this paper.

\section{References}

1. Adams, R. A. and Fournier, J. J. F., Sobolev Spaces, 2nd ed., Pure and Applied Mathematics, Elsevier/Academic Press, Amsterdam, 2003.

2. Agmon, S., Lectures on Elliptic Boundary Value Problems, Van Nostrand, Princeton, NJ, 1965.

3. Axler, S., Bourdon, P. and Ramey, W., Harmonic Function Theory, 2nd ed., Graduate Texts in Mathematics 137, Springer, New York, 2001.

4. Bjerhammar, A. and Svensson, L., On the geodetic boundary-value problem for a fixed boundary surface - satellite approach, Bull. Géod. 57 (1983), 382-393.

5. Boutet De Monvel, L., Boundary problems for pseudo-differential operators, Acta Math. 126 (1971), 11-51.

6. Chazarain, J. and Piriou, A., Introduction à la théorie des équations aux dérivées partielles linéaires, Gauthier-Villars, Paris, 1981.

7. Čunderlík, R., Mikula, K. and Mojzeš, M., Numerical solution of the linearized fixed gravimetric boundary-value problem, J. Geod. 82 (2008), 15-29.

8. Egorov, Ju. V., Subelliptic operators, Uspekhi Mat. Nauk 30 (1975), 57-114, 30 (1975), 57-104 (in Russian); English translation: Russ. Math. Surv. 30 (1975), 59-118, 30 (1975), 55-105.

9. Egorov, Ju. V. and Kondratev, V. A., The oblique derivative problem, Math. USSR, Sb. 7 (1969), 139-169.

10. Fujiwara, D. and Uchiyama, K., On some dissipative boundary value problems for the Laplacian, J. Math. Soc. Japan 23 (1971), 625-635.

11. Guan, P. and Sawyer, E., Regularity estimates for the oblique derivative problem, Ann. of Math. (2) 137 (1993), 1-70.

12. Holota, P., Coerciveness of the linear gravimetric boundary-value problem and a geometrical interpretation, J. Geod. 71 (1997), 640-651.

13. Hörmander, L., Pseudodifferential operators and non-elliptic boundary problems, Ann. of Math. (2) 83 (1966), 129-209.

14. Hörmander, L., Pseudo-differential operators and hypoelliptic equations, in Singular Integrals, Proc. Sympos. Pure Math. X, pp. 138-183, Amer. Math. Soc., Providence, RI, 1967.

15. Hörmander, L., Subelliptic operators, in Seminar on Singularities of Solutions of Linear Partial Differential Equations, Ann. of Math. Stud. 91, pp. 127-208, Princeton Univ. Press, Princeton, New Jersey, 1979.

16. Koch, K. R. and Pope, A. J., Uniqueness and existence for the geodetic boundaryvalue problem using the known surface of the Earth, Bull. Géod. 46 (1972), $467-476$.

17. Melin, A. and Sjöstrand, J., Fourier integral operators with complex phase functions and parametrix for an interior boundary value problem, Comm. Partial Differential Equations 1 (1976), 313-400. 
18. Melin, A. and SJÖTrAnd, J., A calculus for Fourier integral operators in domains with boundary and applications to the oblique derivative problem, Comm. Partial Differential Equations 2 (1977), 857-935.

19. Poincaré, H., Leçons de méchanique céleste, Tome III, Théorie des marées, GauthierVillars, Paris, 1910.

20. Rempel, S. and Schulze, B.-W., Index Theory of Elliptic Boundary Problems, Akademie-Verlag, Berlin, 1982.

21. Schrohe, E., A short introduction to Boutet de Monvel's calculus, in Approaches to Singular Analysis, Oper. Theory Adv. Appl. 125, pp. 85-116, Birkhäuser, Basel, 2001.

22. Seeley, R. T., Singular integrals and boundary value problems, Amer. J. Math. 88 (1966), 781-809.

23. Smith, H., The subelliptic oblique derivative problem, Comm. Partial Differential Equations 15 (1990), 97-137.

24. TAIra, K., Sur le problème de la dérivée oblique II, Ark. Mat. 17 (1979), 177-191.

25. TAIRA, K., Un théorème d'existence et d'unicité des solutions pour des problèmes aux limites non-elliptiques, J. Funct. Anal. 43 (1981), 166-192.

26. Taira, K., Diffusion Processes and Partial Differential Equations, Academic Press, San Diego, New York, London, Tokyo, 1988.

27. Taira, K., Semigroups, Boundary Value Problems and Markov Processes, 2nd ed., Springer Monographs in Mathematics, Springer, Heidelberg, 2014.

28. TAIrA, K., Analytic Semigroups and Semilinear Initial-Boundary Value Problems, 2nd ed., London Mathematical Society Lecture Note Series 434, Cambridge University Press, Cambridge, 2016.

29. TAIRA, K., Analytic semigroups for the subelliptic oblique derivative problem, J. Math. Soc. Jpn. 69 (2017), 1281-1330.

30. TAYlor, M., Pseudodifferential Operators, Princeton Mathematical Series 34, Princeton University Press, Princeton, NJ, 1981.

31. Treves, F., A new method of the subelliptic estimates, Comm. Pure Appl. Math. 24 (1971), 71-115.

32. Winzell, B., The oblique derivative problem, I. Math. Ann. 229 (1977), 267-278.

\author{
Kazuaki Taira \\ Institute of Mathematics \\ University of Tsukuba \\ Tsukuba 305-8571 \\ Japan \\ Tel.: +81-29-853-4235 \\ taira@math.tsukuba.ac.jp
}

Received March 16, 2016 